\title{
Charge State Distribution Shifting of Protein Ions Observed in Matrix-Assisted Laser Desorption Ionization Mass Spectrometry
}

\author{
J. Zhou and T. D. Lee \\ Beckman Research Institute of the City of Hope, Duarte, California, USA
}

\begin{abstract}
By using a new sample preparation method for matrix-assisted laser desorption ionization, a significant shift to lower mass-to-charge values can be obtained for many protein samples. The sample preparation technique involves the creation of a thin film of protein-doped $\alpha$-cyano-4-hydroxycinnamic acid (CHCA) matrix formed in the presence of glycerol on top of a previously deposited pad of CHCA matrix. The higher charge states were not observed if the laser power was significantly above the threshold needed to produce protein molecular ions. Similar spectra were observed when samples were prepared in the presence of urea. The phenomenon was specific for the CHCA matrix because no effects were observed when sinapinic acid (3,5-dimethoxy-4-hydroxy-trans-cinnamic acid) and 2-(4-hydroxyphenylazo) benzoic acid matrices were used with the new sample preparation method. (/ Am Soc Mass Spectrom 1995, 6, 1183-1189)
\end{abstract}

$\mathrm{M}$ atrix-assisted laser desorption-ionization (MALDI) [1] and electrospray (ES) [2] mass spectrometry have become two very powerful techniques in the analysis of proteins. Accurate mass measurements for proteins up to $300 \mathrm{ku}$ can be obtained with these two ionization methods. Electrospray is a soft ionization technique that produces multiply charged ions from proteins in solution [2]. Many laboratories have reported the investigation of protein conformational changes as evidenced by changes in charge state distributions in ES mass spectra [3-9]. Such changes in charge state distributions of proteins in the gas phase have been obtained by changing protein solution conditions such as $\mathrm{pH}$ [3], organic solvent content $[4]$, and temperature $[5,6]$. The lower and higher charge state distributions obtained generally are interpreted to be derived from folded and unfolded conformations of the protein in solution. Elastic collisions carried out in the collision cell of a triple sector quadrupole mass spectrometer were found to have dramatic effects on the appearance of the two charge state distributions in the mass spectra of apomyoglobin and other proteins [7]. Collisions of low energy multiply charged ions with various target gases have been reported to shift the distribution of protein ions to higher mass-to-charge values $[8,9]$.

Unlike ES, where gas-phase sample ions are produced directly from the solution phase, standard MALDI procedures produce ions from solid matrices.

Address reprint requests to Dr. Terry D. Lee, Division of Immunology, Beckman Research Institute of the City of Hope, 1450 E. Duarte Road, Duarte, CA 91010.
The MALDI process favors the production of ions with few charges (high mass-to-charge ratios). Some matrices, such as $\alpha$-cyano-4-hydroxycinnamic acid (CHCA) [10] when used with ultraviolet lasers (337 and $355 \mathrm{~nm}$ ) and caffeic acid when used with infrared lasers $(2.94$ and $10.6 \mathrm{~mm}$ ) [11, 12], yield multiply charged species. However, the charge states observed are much lower than those observed with ES. Liquid matrices such as glycerol have been used for MALDI, but apparently yield mostly singly charged species [11, 12]. We are unaware of any published explanation for why the CHCA matrix yields multiply charged species when other matrixes do not. The chemical reactions that take place during desorption would vary dependent on the matrixes. Additionally, experiments have shown that protein ions undergo numerous collisions during the MALDI process that lead to relatively high kinetic energy and internal excitation [13-15]. Multiply charged species would undergo higher energy collisions during the extraction process in a time-of-flight ion source and may be subject to varying degrees of charge stripping.

Recently, Xiang and Beavis [16] reported that a thin polycrystalline film, which consisted of microcrystals of protein-doped matrix, could quickly form on a previously deposited layer of the same matrix. The sample targets formed in this way are more uniform and yield more intense and reproducible spectra than targets produced by the conventional dried-droplet method. The film strongly adheres to the substrate so that it can be washed more easily and samples can be prepared in the presence of high concentrations of glycerol and even 6-M urea. During experiments with 
our own version of the Beavis method with the CHCA matrix, we observed significant increases in the intensities of higher charge state species when the polycrystalline film was formed in the presence of glycerol. In the case of phophorylase a, there was a bimodal charge state distribution. This phenomenon has been studied in some detail and we report our findings in this article.

\section{Experimental}

Experiments were conducted in a Kratos Compact MALDI III reflectron MALDI time-of-flight mass spectrometer (Shimadzu, Columbia, MD). All mass spectra were collected in the linear mode without use of the reflectron. The stainless steel sample slide contains 20 rectangular sample stage wells $(2 \mathrm{~mm} \times 1.5 \mathrm{~mm})$. The focused laser spot is fixed and the sample stage is moved in the horizontal direction perpendicular to the axis of the flight tube. Thus, only a thin strip of the sample well was irradiated by the laser beam. Spectra reported are an average of 100 laser shots over the length of the sample well. The incident laser irradiance can be adjusted with a continuous laser attenuator controlled by the computer. The computer reports only the percentage of the full laser power rather than the absolute value of laser power density. The sample slide was floated at $20 \mathrm{kV}$. A nitrogen laser $(337 \mathrm{~nm})$ was used for desorption, and an electron multiplier was used for ion detection. There are no deflection plates to deflect light matrix ions.

The $\alpha$-cyano-4-hydroxycinnamic acid (CHCA) (Aldrich, Milwaukee, WI) was prepared as a saturated solution at room temperature $\left(\sim 23^{\circ} \mathrm{C}\right)$ in $0.1 \%$ trifluoroacetic acid (TFA) and acetonitrile $(1: 1 \mathrm{v} / \mathrm{v})$. The fucose (Aldrich) solution was prepared in the same solvent at a concentration of $10 \mathrm{~g} / \mathrm{L}$. Bovine serum albumin (BSA) and phosphorylase a [with 60\% $\beta$ glycerophosphate and ethylenediaminetetraacetic acid (EDTA); Sigma, St. Louis, MO] were dissolved in $0.1 \%$ TFA at concentrations of 5 and $2 \mathrm{~g} / \mathrm{L}$. Antibody MT 84.66 (from our own laboratory) was also in $0.1 \%$ TFA at a concentration of $14 \mathrm{~g} / \mathrm{L}$.

Because the wells on the sample stage for the Kratos MALDI III were recessed, the initial layer of matrix was thicker than that used by Xiang and Beavis [16]. Matrix solution $(0.5-1.0 \mu \mathrm{L})$ was placed on the sample stage and allowed to dry. The process was repeated until about $4 \mu \mathrm{L}$ of sample solution had been added and a solid matrix pad completely filled the well. A microscope slide was put on the top of the matrix, pressed, and smeared with one finger. Loose matrix powders were removed by tapping the sample slide, which left fine matrix powders that adhered to the stainless steel substrate. Samples applied to the matrix pad could be washed by dipping the slide in roomtemperature water for over $2.0 \mathrm{~min}$ without loss of the matrix pad. Protein solutions (a few picomoles total amount) were mixed with matrix solution and applied to the matrix pad and allowed to dry completely. For some experiments, the protein solution was mixed with a solution that contained matrix and fucose $(2: 1 \mathrm{v} / \mathrm{v})$.

When glycerol was added to the sample-matrix solution ( $20 \%$ by volume), there was no obvious change in the sample drop volume after a few minutes and the remaining liquid was removed from the pad by dipping the slide in room temperature water for $2 \mathrm{~min}$. Consequently, there was no way to estimate the amount of sample actually deposited on the pad. For the measurements of BSA with urea, the BSA solution was mixed with the matrix dissolved in 6-M urea and acetonitrile $(1: 1 \mathrm{v} / \mathrm{v})$ and $1.0 \mu \mathrm{L}$ of the solution was deposited onto the matrix pad. After $1 \mathrm{~min}$, the target slide was dipped in water for $2 \mathrm{~min}$ to wash away the remaining liquid. Phosphorylase a prepared the same way gave a very weak spectrum. Better spectra were obtained when the phosphorylase a was dissolved in $0.2-\mathrm{M}$ urea in water and mixed with the matrix solution (in $0.1 \%$ aqueous TFA and acetonitrile, $1: 1 \mathrm{v} / \mathrm{v}$ ), deposited on the matrix pad, completely dried, and then dipped in water for $2 \mathrm{~min}$.

Reduced and alkylated phosphorylase a was prepared by dissolving $100 \mu \mathrm{g}$ of phosphorylase $\mathrm{a}$ in $500 \mu \mathrm{L}$ of $6-\mathrm{M}$ quanidine hydrochloride, $25-\mathrm{mM}$ tris(hydroxymethyl)aminomethane, and 1-mM EDTA at $\mathrm{pH} 8.5$. A $25-\mu \mathrm{L}$ solution of $\beta$-mercaptoethanol in water was added and the reaction solution was incubated in the dark under argon for $2 \mathrm{~h}$. Then $25 \mu \mathrm{l}$ of 4 -vinylpyridine was added, the sample was incubated another $2 \mathrm{~h}$, and then stored at $5{ }^{\circ} \mathrm{C}$ until it was purified by gel filtration by using a Sephadex G-25 superfine column that eluted with $0.1 \%$ aqueous TFA.

Circular dichroism (CD) spectra of BSA were measured at a concentration of $0.1 \mathrm{~g} / \mathrm{L}$ in solutions of $50 \%$ acetonitrile-water, and with added glycerol $(20 \%$ by volume) or urea ( $6 \mathrm{M}$ ). The $\mathrm{CD}$ spectrometer used was a Jasco J600 spectropolarimeter (Jasco Inc., Easton, MD).

\section{Results}

The effect of the use of glycerol in the sample preparation was evident in the MALDI analysis of BSA (Figure 1). With a laser power setting of $30 \%$, there was only a minor difference in the spectra obtained via the dried-droplet method on the bare sample stage (Figure 1A) or on the matrix pad (Figure 1B). However, when the sample was prepared as a thin film from a solution that contained $20 \%$ glycerol on the matrix pad (Figure 1C), there was a significant increase in the relative intensities of the higher charge states $(5+$, $6+, 7+, 8+$, and $9+)$ and a corresponding decrease in the relative intensities of the lower charge states $(1+$ and $2+)$. Additionally, absolute signal intensities were higher for the sample prepared with glycerol with a corresponding improvement in the signal-to-noise ratio. The actual spectra for the samples prepared with and without glycerol are given in Fig- 

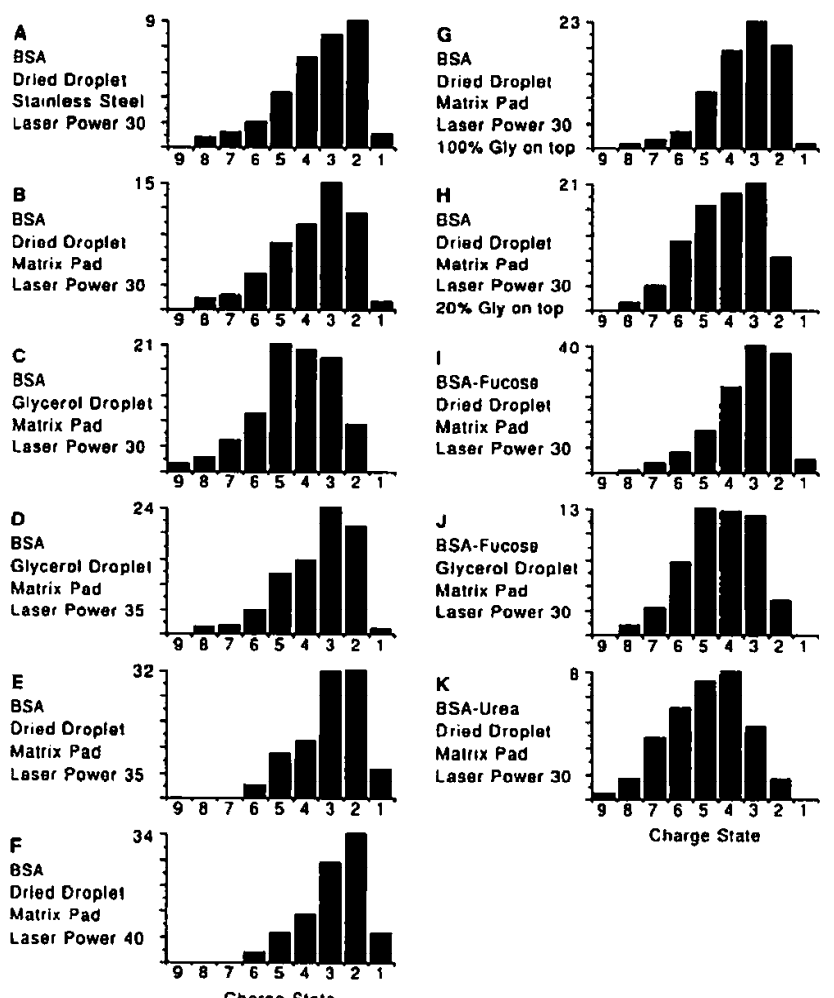

Figure 1. Graph of the relative intensities of various charge states observed in the positive ion MALDI spectra of BSA obtained near threshold (30\% full laser power) from (A) dried BSA-CHCA droplet on a stainless steel substrate; (B) BSA-doped CHCA film on a CHCA matrix pad; (C) the corresponding protein-doped matrix film formed in the presence of glycerol; (D) sample preparation the same as (C), but with 35\% full laser power; (E) sample preparation the same as (B) but with $35 \%$ full laser power; (F) sample preparation the same as (B) but with $40 \%$ full laser power; (G) sample preparation the same as (B) with subsequent addition of glycerol and then a water wash; $(\mathbf{H})$ sample preparation the same as (B) with subsequent addition of $20 \%$ aqueous glycerol followed by a water wash; (I) sample prepared as a BSA-doped CHCA matrix and fucose co-matrix $(2: 1 \mathrm{v} / \mathrm{v})$ film on a CHCA matrix pad; $(J)$ sample preparation same as for (I) only film is formed from the CHCA matrix-fucose solution that contained $20 \%$ glycerol; (K) sample deposited on a CHCA matrix pad from a solution of BSA and CHCA matrix in 6-M aqueous urea and acetonitrile $(1: 1 \mathrm{v} / \mathrm{v})$. All graphs are normalized to the most abundant charge state observed. The number at the top of the $y$-axis is the absolute value (millivolts) of the most abundant charge state.

ure $2 \mathrm{~A}$ and $\mathrm{B}$. When the laser power was increased to $35 \%$ for the sample prepared by using glycerol on the matrix pad, the shift in charge state distribution disappeared, although the improvement in the signal-tonoise ratio was retained (Figure 1D). Further increases in laser power (data not shown) result in increased signal intensities, but at the expense of degrading the resolution (broad peaks) and with no additional change in the charge state distribution. For samples prepared in the absence of glycerol, there was a slight shift in the charge state distribution as the laser power was increased from 30 (Figure 1B) to 35 (Figure 1E) to $40 \%$ (Figure 1F). This is a common phenomenon observed
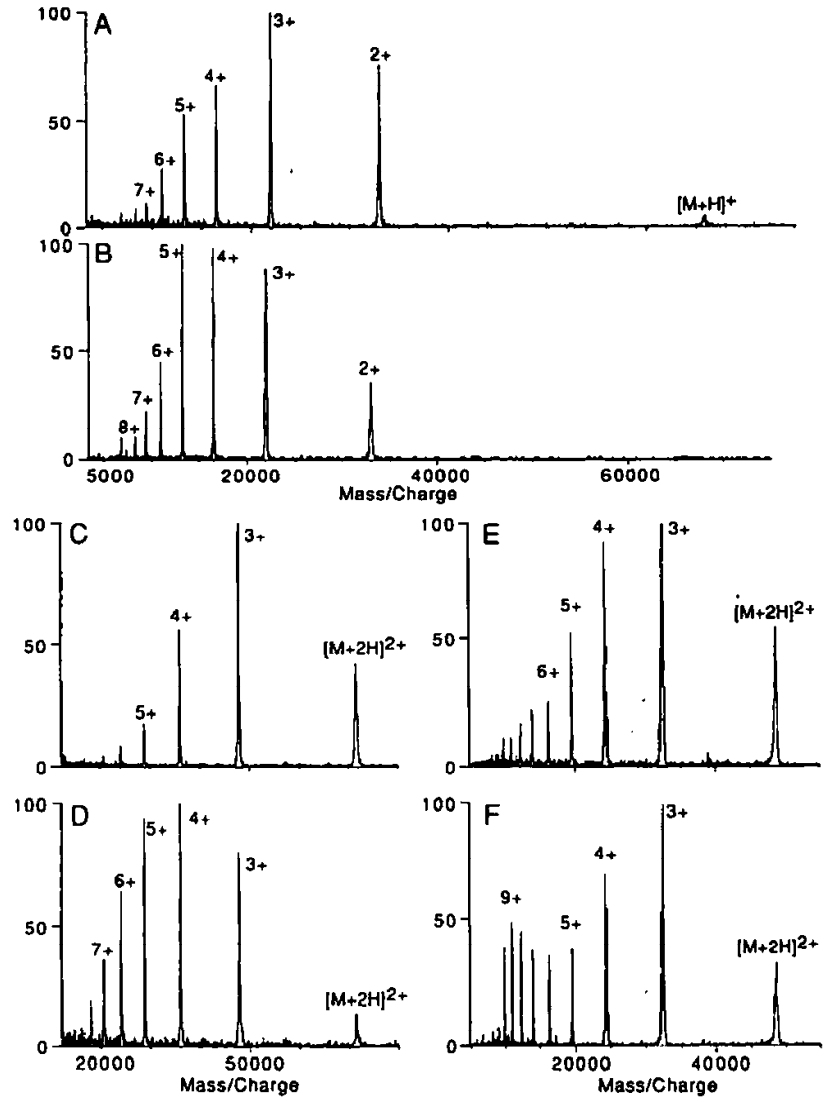

Figure 2. Comparison of the effect of glycerol on the charge state distribution in positive ion MALDI spectra of three proteins. BSA-matrix layer formed $(A)$ via the dried-droplet method and (B) from a $20 \%$ glycerol droplet (30\% full laser power). Antibody MT 84.66 formed (C) via the dried-droplet method, and (D) from a $20 \%$ glycerol droplet (33\% full laser power). Phosphorylase a formed (E) via the dried-droplet method and (F) from a $20 \%$ glycerol droplet ( $40 \%$ full laser power). All samples were deposited on a CHCA matrix pad.

in many labs, but the charge state shift was much more pronounced with samples prepared by using glycerol.

Further experiments were performed to probe the nature of the interaction of glycerol with the sample and matrix. First samples were prepared as dried droplets on a matrix pad. A bead of glycerol was placed on top of the sample and left for several minutes before the slide was dipped in water and dried. The resulting spectrum (Figure 1G) was similar in intensity and charge state distribution to that for a sample not treated with glycerol (compare with Figure 1B). When the experiment was repeated by using a bead of $20 \%$ aqueous glycerol (Figure $1 \mathrm{H}$ ), a lesser amount of charge shift was noted compared to sample films formed in the presence of glycerol. The $3+$ charge state still had the maximum intensity, but there was a relative decrease in the intensity of the $2+$ charge state and increases in the $4+$ to $8+$ charge states. Analogous experiments also were done by using aqueous glycerol solutions that contained 1,5 , and $10 \%$ glycerol. A similar small degree of charge shift 
was noted for most samples compared to samples not treated with glycerol. However, there was no trend consistent with differing amounts of glycerol and results were much less consistent than when the glycerol was added with the sample and matrix solution.

Various sugars have been used with normal MALDI matrices to improve the quality of MALDI spectra for peptides and proteins $[17,18]$. With the thought that the charge state shifting phenomenon might be related to the benefits observed with sugar co-matrices, experiments were done to determine the effect of added fucose. The spectrum of BSA ions desorbed at $30 \%$ full laser power from the BSA-doped CHCA and fucose film on a CHCA matrix pad (Figure 1I) differed little from the spectrum obtained without fucose (Figures 1B). If anything, the relative intensities of the $2+$ and $1+$ charge states were enhanced and may indicate a certain amount of charge stripping. When the sample-matrix-fucose film was formed from a solution that contained $20 \%$ glycerol, a significant shift in the charge states was observed (Figure 1J) similar to samples prepared without fucose (Figure 1C).

To determine how general the charge shifting phenomenon is, a number of different proteins were analyzed. Smaller proteins such as apomyoglobin and carbonic anhydrase did not show a significant difference in the charge state distribution when samples were prepared with and without glycerol. Observed absolute intensities are somewhat better for the samples prepared with glycerol (data not shown). The antibody MT 84.66 did exhibit a shift to higher charge states (Figure 2C and D) when prepared from a glycerol solution, but in this instance there was a decrease in the signal-to-noise ratio. With phosphorylase a (Figure $2 \mathrm{E}$ and $\mathrm{F}$ ), the results were very dramatic. For spectra taken near the laser power threshold $(40 \%$ of full laser power) with samples prepared in the absence of glycerol on stainless steel substrate (Figure $3 \mathrm{~A}$ ) and with a CHCA matrix pad (Figures 3B and $2 \mathrm{E}$ ), there was a single envelope of charge states with a maximum at $3+$. For the sample prepared with glycerol (Figures $3 \mathrm{C}$ and $2 \mathrm{~F}$ ) the charge state distribution was bimodal with maxima at $3+$ and $9+$. As with BSA, the relative intensities of the higher charge states decreased significantly when the laser power was increased ( $45 \%$ of full laser power; Figure 3D). The relative intensities of the two distributions were found to change somewhat from sample to sample even under the same laser power.

Phosphorylase a was reduced and alkylated in an attempt to generate a fully denatured form that would accommodate more charges. However, spectra of the reduced and alkylated material required a higher laser power $(45 \%)$ and none of the spectra resembled any of the spectra of the original protein (data not shown). For the spectrum obtained without glycerol, only the $1+, 2+$, and $3+$ charge states had intensities significantly above background noise levels; the $2+$ charge state had twice the relative intensity of the $3+$
A

Phosphorylase a Dried Droplet Stainless Steel Laser Power 40

B Phosphorylase a Dried Droplet Matrix Pad

Laser Power 40

C

Phosphorylase a Glycerol Droplet Matrix Pad

Leser Power 40

D Glycerol Droplet Matrix Pad Laser Power 45

E

Phosphorylase a - Urea

Dried Droplet

Matrix Pad

Laser Power 40

$\mathbf{F}$

Phosphorylase a - Urea

Dried Droplet

Matrix Pad

Laser Power 45
Phosphorylase a
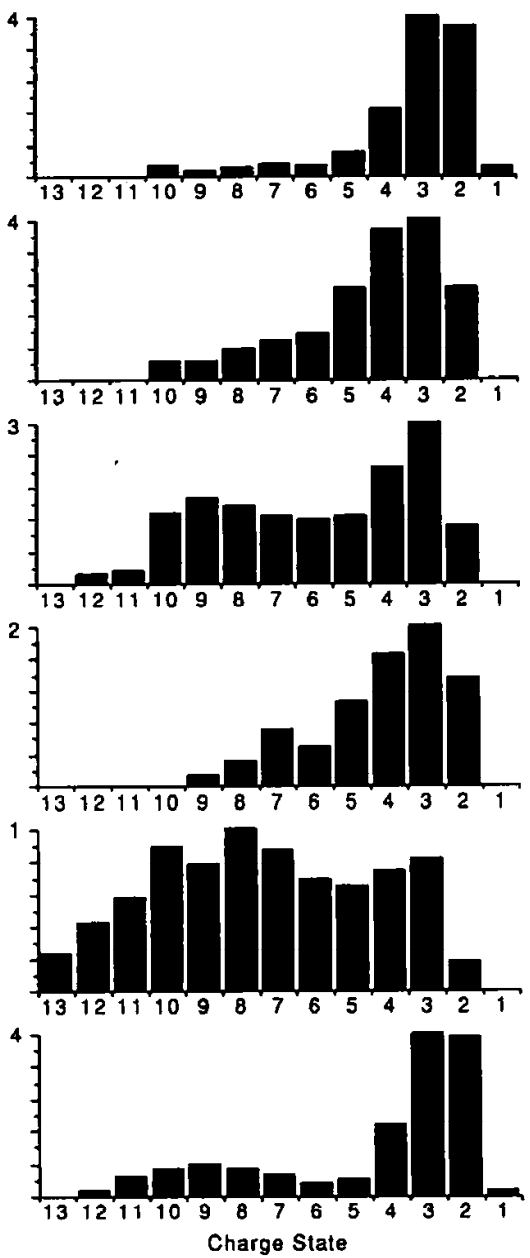

Figure 3. Positive ion MALDI spectra of phosphorylase a obtained near threshold ( $40 \%$ full laser power) from $(\mathrm{A})$ the dried protein-doped CHCA matrix droplet on stainless steel substrate; (B) the protein-doped CHCA film formed on a CHCA matrix pad; (C) the corresponding film formed in the presence of glycerol on the same matrix pad; (D) the same sample preparation as (C) but with $45 \%$ full laser power; (E) sample prepared from a $0.2-\mathrm{M}$ aqueous urea-acetonitrile $(1: 1 \mathrm{v} / \mathrm{v})$ solution that contained the protein and CHCA on a CHCA matrix pad; (F) same sample preparation only with a laser power of $45 \%$.

charge state. For the spectrum obtained with glycerol, the $3+$ and $2+$ charge states were of nearly equal intensity and the signal-to-noise ratio was improved.

The effect of urea as a MALDI matrix modifier also was studied. In solution, urea and glycerol have much different effects on protein structure. Urea is routinely used as a denaturing agent. It disrupts intramolecular forces that hold the protein in its native conformation [19]. Glycerol, on the other hand, is used to stabilize native conformations [20]. The effect of glycerol and urea on the conformation of BSA in solution can be monitored by circular dichroism (CD) spectrometry (Figure 4). In the presence of glycerol the CD spectrum of BSA indicates more alpha helical content compared to the spectrum of BSA in the same solvent without glycerol. In contrast, added urea results in a CD spectrum characteristic of a random coil structure. 


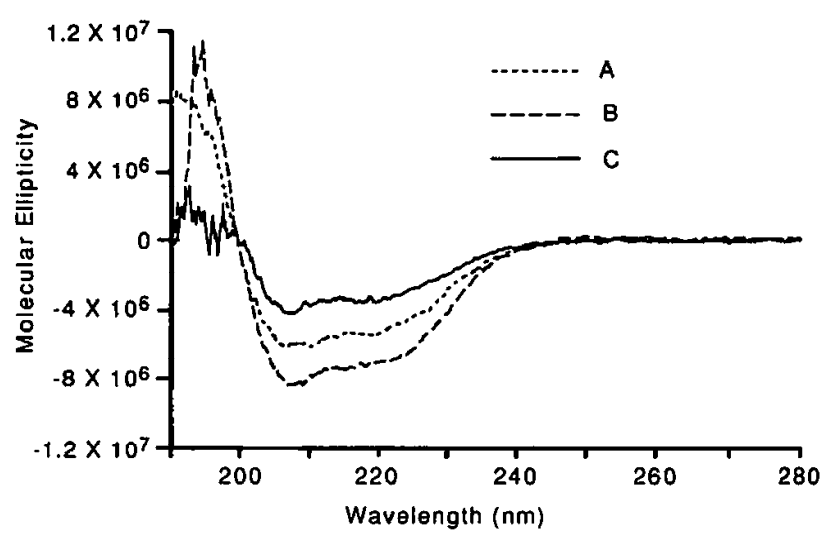

Figure 4. Circular dichroism (CD) spectra of a $0.1-\mathrm{g} / \mathrm{L}$ BSA solution of (A) acetonitrile-water $(1: 1$ by volume), (B) in the same solvent that contains $20 \%$ glycerol, and (C) $20 \% 6-\mathrm{M}$ urea by volume.

For the MALDI spectra, the solution that contained the matrix, urea, and protein was allowed to adsorb on the matrix pad for $1 \mathrm{~min}$ before washing with water. The spectrum obtained for BSA (Figure 1K) showed charge shift similar to that obtained with glycerol, although the signal-to-noise ratio of the spectra was not as good. The spectrum obtained for phosphorylase a at threshold laser powers with a lesser amount of urea ( $0.2 \mathrm{M}$ versus $6 \mathrm{M}$; Figure $3 \mathrm{E}$ ) exhibited the same bimodal charge state distribution, although the spectrum was very weak. With a small increase in laser power, the bimodal distribution still was observed, but the relative amounts of each distribution had changed dramatically (Figure 3F). Unfortunately, it was not possible to use CD spectrometry to monitor protein conformation in the matrix solutions used to obtain the MALDI spectra because the matrix absorbs strongly in the wavelength range that $C D$ measurements are made.

Measurements performed via the glycerol preparation method also were carried out with sinapinic acid and 2-(4-hydroxyphenylazo)benzoic acid (HABA) matrices. However, no corresponding charge state shift of protein ions was observed with these two matrices. Both these matrices required higher laser power densities than the CHCA matrix to achieve the threshold level for protein desorption.

\section{Discussion}

The production of ions with higher numbers of charges when prepared in the presence of glycerol by using the CHCA matrix is a reproducible phenomenon for proteins larger than $50 \mathrm{ku}$. Understanding the nature of this phenomenon is complicated by the complex nature of the MALDI sample preparation and desorption process. Various researchers have shown that proteins are incorporated in matrix crystals in a nonrandom fashion [21, 22]. However, details about protein conformation and the extent that solvent and electrolyte components are included along with the protein are not known. Certainly there is some exclusion of sample impurities as evidenced by the ability to wash away salts and other contaminants that tend to interfere with the analysis. Although there is no direct evidence in the spectra, our experiments indicate that glycerol is intimately associated with the protein in the CHCA crystal structure. Attempts to deposit glycerol as a layer on the sample matrix after it was formed gave inconsistent results with a more limited amount of charge state shift. No method was found to add glycerol in such a way as to preclude intimate mixing with the sample and matrix. Consistent results always were obtained when glycerol was present when the film of matrix and sample was initially formed.

Urea also was found to shift the observed çharge state distribution to higher values. Like glycerol, urea is a small, polar molecule. However, there is a considerable difference between glycerol and urea in the nature of their interactions with proteins. High concentrations (1-6 M) of urea are routinely used by protein chemists to denature proteins. The small urea molecules effectively displace water from the interior of large protein structures and disrupt intraprotein hydrogen bonds, which causes the protein to unfold and assume a more extended conformation [19]. It is reasonable to assume that these extended conformations would be incorporated into the MALDI matrix crystals. Proteins with extended conformations might be expected to accumulate more charges during the desorption process analogous to what is observed via electrospray. Glycerol on the other hand is used to solubilize proteins and preserve their native conformations and activities in solution [20]. It was surprising to us that spectra obtained by using glycerol would show the same degree of multiple charging as spectra obtained by using urea when, presumably, initial conformations in solution as indicated by the $C D$ spectra would be very different. The effect on protein conformation of the high concentration of the matrix material in the sample solution and constraints imposed by the crystallization process are not known. One possibility is that in the absence of either glycerol or urea, the protein is forced to assume a more compact, nonnative conformation that limits the number of charges acquired by the sample during the desorption process. Another possibility is that glycerol and urea similarly influence chemical reactions during the desorption process and perhaps make more protons available to charge protein ions. Any comprehensive explanation of the observed phenomenon must take into account the strong dependence on laser power levels.

Spectra obtained for phosphorylase a by using glycerol or urea appear to have two distinct charge state distributions. This bimodal distribution persists at higher laser power levels, but the intensity of the higher charge distribution decreases dramatically relative to the lower charge distribution (compare Figure $3 C, D$ and $3 E, F$ ). These results argue strongly for two distinct populations of ions, one of which is very 
sensitive to laser power levels. The charge state shift observed for other proteins also may result from two distinct distributions that happen to overlap in such a way that the bimodal distribution is not observed. Although it may be possible to relate these changes to some aspect of protein structure in the crystal, it is very unlikely that charge state distributions observed in MALDI will provide any useful information about the state of proteins in solution.

Given the resolution and mass accuracy of our measurements for these large molecules, we cannot rule out the possibility that the observed ions carry adducts of glycerol or urea with them. It has been established in our lab (unpublished results) and others that large protein ions produced by MALDI have greater apparent masses relative to lower molecular weight internal calibrants. This is due at least in part to an energy deficit that results from greater numbers of collisions for larger structures in the acceleration region of a linear time-of-flight mass spectrometer [14, 15]. However, there also may be increased numbers of adducts with ions and small molecules that are unresolved on low resolution time-of-flight instruments. Further insight on this aspect of the desorption process possibly could be obtained from studies on high resolution instruments such as magnetic sector or Fourier transform ion cyclotron resonance (FTICR) mass spectrometers provided these highly charged ions can be produced and trapped in these instruments. The use of matrices such as CHCA and the glycerol preparation technique that we have described may provide the means to produce high mass ions by MALDI with mass-to-charge ratios more easily accessible to magnetic sector and FTICR instruments. Published studies on MALDI that used magnetic sector and Fourier transform mass spectrometry instruments have used matrices such as sinipinic acid and 2,4-dihydroxybenzoic acid and only singly charged species were observed [23-27].

Sugars such as fructose have been used as a co-matrix in MALDI analyses on FTICR instruments [17, 26, 28]. Decomposition of the carbohydrate co-matrix during the desorption process is thought to provide a dense cloud of gas. Collisions of the sample ions with the neutral gas molecules serve to cool the ions, which reduces the excess energy imparted during the desorption event. The cooled sample ions exhibit less metastable decomposition and are easier to trap in the FTICR analyzer cell. The use of fucose as a co-matrix in MALDI time-of-flight (TOF) analyses of peptide mixtures has been reported to provide spectra with greater abundance, higher resolution, and less discrimination among mixture components [18]. In most MALDI TOF mass spectrometers, ions are formed in an accelerating electric field. Collisions with neutral gas molecules would be expected to result in transfer of translational energy to vibrational modes and thus increase the probability of fragmentation. We have found that the addition of fucose to the matrix has no significant effect on the charge state distribution. Samples prepared with and without glycerol in the presence of added fucose showed the same shift in the charge state distribution as the same samples prepared in the absence of fucose. Thus, although fucose and glycerol share common structural elements, their effects on charge state distribution are entirely different.

Thus far, the laser power level has the most significant effect on the charge shifting phenomenon. The highly charged species are always most intense when the laser power is near the threshold level and intensities decrease precipitously, as the laser power is increased (compare Figure $1 C$ and D, Figure $3 C$ and D, and Figure $3 \mathrm{E}$ and $\mathrm{F}$ ). Further increases in laser power result in loss of resolution due to increased metastable decay, but with only small changes in the relative intensities of the remaining charge states. We interpret this as evidence for an initial state of the protein ions that is easily disturbed by excess laser power with resultant loss of charge. It is significant that the multiple charging phenomenon is observed only in the CHCA matrix, which has a much lower laser power threshold for protein ion desorption than either sinapinic acid or HABA.

\section{Conclusion}

The shift of charge state distribution to higher charge values was observed for large proteins ( $>50 \mathrm{ku}$ ) when samples were prepared as thin films from solutions that contained glycerol. The degree of shift was strongly dependent on laser power levels and was observed near the irradiance threshold for protein ion formation. Samples prepared in the presence of urea showed a similar increase in the intensity of more highly charged ions. Given the known differences between glycerol and urea on protein conformations in solution, it is unlikely that charge state distributions in MALDI spectra can be correlated to protein conformations in solution. The addition of fucose to the matrix had no significant effect on the charge state distributions observed either in the presence or absence of glycerol. Thus, the phenomenon appears to be unrelated to increased collisions thought to occur when a sugar co-matrix is used. No corresponding charge state shift could be obtained with sinapinic acid and HABA matrices. The use of the CHCA matrix and the described sample preparation technique may make it possible to obtain MALDI spectra for large proteins on magnetic sector and FTICR mass spectrometers with limited mass range capabilities.

\section{Acknowledgments}

We thank Dr. John Shively for valuable discussions and for providing the antibody sample, Mark Sherman for assistance with the CD spectrometry, and Dr. Kristine Swiderek for providing the reduced and alkylated phosphorylase a sample. This work was supported in part by Department of Health and Hu- 
man Services, Public Health Service grants HG00863 and CA33572.

\section{References}

1. Hillenkamp, F.; Karas, M.; Beavis, R. C.; Chait, B. T. Anal. Chem. 1991, 63, 1193A-1203A.

2. Fenn, J. B.; Mann, M.; Meng, C. K.; Wong, S. F.; Whitehouse, C. M. Science 1989, 246, 64-71.

3. Chowdhury, S. K.; Katta, V.; Chait, B. T. I. Am. Chem. Soc. 1990, 112, 9012-9013.

4. Loo, J. A.; Loo, R. R. O.; Udseth, H. R.; Edmonds, C. G.; Smith, R. D. Rapid Conmmun. Mass Spectrom. 1991, 5, 101-105.

5. Mirza, U. A.; Cohen, S. L.; Chait, B. T. Allal. Chem. 1993, 65 , $1-6$.

6. Leblanc, J. C. Y.; Beuchemin, D.; Siu, K. W. M.; Guevremont, R.; Berman, S. S. Org. Mass Spectrom. 1991, 26, 831-839.

7. Cox, K. A.; Julian, R. K., Jr.; Cooks, R. G.; Kaiser, R. E., Jr. J. Am. Soc. Mass Spectrom. 1993, 5, 127-136.

8. McLuckey, S. A.; Van Berkel, G. J.; Glish, G. L. J. Am. Chem. Soc. 1990, 112, 5668-5670.

9. Ikonomou, M. G.; Kebarle, P. Int. I. Mass Spectrom. Ion Processes 1992, 117, 283-298.

10. Beavis, R. C.; Chaudhary, T.; Chait, B. T. Org. Mass Spectrom. 1992, 27, 156-158.

11. Overberg, A.; Karas, M.; Bahr, U.; Kaufmann, R.; Hillenkamp, F. Rapid Commun. Mass Spectrom. 1990, 4, 293-296.

12. Overberg, A.; Karas, M.; Hillenkamp, F. Rapid Conmun. Mass Spectrom. 1991, 5, 128-131.

13. Beavis, R. C.; Chait, B. T. Chem. Phys. Lett. 1991, 181, 479-484.
14. Zhou, J.; Ens, W.; Standing, K. G.; Verentchikov, A. Rapid Commun. Mass Spectrom. 1992, 6, 671-678.

15. Ens, W.; Spicer, V.; Standing, K. G.; Zhou, J. Org. Mass Spectrom. 1993, 28, 1430-1434.

16. Xiang, F.; Beavis, R. C. Rapid Contmun. Mass Spectrom. 1994, 8, 199.

17. Koster, C.; Castoro, J. A.; Wilkins, C. L. J. Am. Chem. Soc. 1992, 114, 7572-7574.

18. Billeci, T. M.; Stults, J. T. Anal. Chem. 1993, 65, 1709-1716.

19. Prakash, V.; Loucheux, C.; Scheufele, S.; Gorbunoff, M. J.; Timasheff, S. N. Arch. Biochen. Biophys. 1981, 210, 455-464.

20. Timasheff, S. N.; Arakawa, T. In Protein Structure, a Practical Approach; Creighton, T. E., Ed.; IRL Press: New York, 1989; p. 331-345.

21. Strupat, K.; Karas, M.; Hillenkamp, F. Int. J. Mass Spectrom. Ion Processes 1991, 111, 89-102.

22. Beavis, R. C.; Bridson, J. N. J. Phys. D 1993, 26, 442-447.

23. Li, Y. Z.; Mciver, R. T.; Hunter, R. L. Anal. Chem. 1994, 66, 2077-2083.

24. Solouki, T.; Gillig, K. J.; Russell, D. H. Anal. Chem. 1994, 66, 1583-1587.

25. Hill, J. A.; Annan, R. S.; Biemann, K. Rapid Commun. Mass Spectrom. 1991, 5, 395-399.

26. Castro, J. A.; Koster, C.; Wilkins, C. Rapid Commun. Mass Spectrom. 1992, 6, 239-241.

27. Sheng, L. S.; Covey, J. E.; Shew, S. L.; Winger, B. E.; Campana, J. E. Rapid Commun. Mass Spectrom. 1994, 8, 498-500.

28. Solouki, T.; Gillig, K. J.; Russell, D. H. Rapid Commun. Mass Spectrom. 1994, 8, 26-31. 